\title{
PULMONARY PLACENTAL TRANSMOGRIFICATION: DIAGNOSIS AND TREATMENT
}

\author{
Gregory R. Brevetti, MD, Carolyn Clary-Macy, RN, and David M. Jablons, MD, San Francisco, Calif
}

A case of giant bullous disease with pulmonary transmogrification is described in which the bulla was initially mistaken for a pneumothorax. This report reviews the possible pitfalls in the diagnosis of this rare histologic subtype of bullous emphysema, which has been described in only 8 patients. The patient course including successful surgical resection and management options are reviewed.

Emphysema occurs most commonly in smokers and patients with $\alpha_{1}$-antitrypsin deficiency. A rare congenital form of giant bullous emphysema was described in 1979 and termed pulmonary placental transmogrification. ${ }^{1}$ It is rare and has been described once in the surgical literature. ${ }^{2}$

Clinical summary. The patient is a 39-year-old white woman who underwent a left modified radical mastectomy for ductal carcinoma in situ with immediate reconstruction with a saline implant. A preoperative chest $\mathrm{x}$-ray film revealed a "hyperinflated right lung, with decreased vascular markings." At the time of subpectoral prosthetic implantation, the pleura was violated and a thoracostomy tube placed. A postoperative chest radiograph revealed good expansion on the left side and what was believed to be a right pneumothorax. A thoracostomy tube was placed in the right side of the chest. She was then referred for definitive management after the left pneumothorax resolved but the right-sided abnormality, which was, in reality, a giant bulla persisted (Fig 1). A computed tomographic scan revealed a giant right upper lobe bulla with partial right middle and lower lobe inflation (Fig 2). The mediastinal structures were shifted to the left, but the patient tolerated this well. She underwent video-assisted thoracoscopic surgery with successful staple resection of this giant bulla. On postoperative day 3 a small air leak had sealed. On postoperative day 4, however, she had a new, significant air leak that persisted and culminated in re-exploration on postoperative day 7 . At thoracotomy, a very small, previously obscured bulla originating from the lower lobe was found to have a 2-mm hole. The bulla was stapled and a generous parietal pleurectomy was performed. Her subsequent recovery was uneventful and she was discharged 3 days later with a fully expanded right lung.

From the Division of Cardiothoracic Surgery, University of California-San Francisco, San Francisco, Calif.

Received for publication April 13, 1999; accepted for publication June 29, 1999.

Address for reprints: Gregory R. Brevetti, MD, Division of Cardiothoracic Surgery, University of California-San Francisco, 505 Parnassus Ave, Moffitt 593, Box 0118, San Francisco, CA 941430118.

J Thorac Cardiovasc Surg 1999;118:966-7

Copyright (C) 1999 by Mosby, Inc.

$0022-5223 / 99 \$ 8.00+0 \quad \mathbf{1 2 / 5 4 / 1 0 1 1 9 9}$

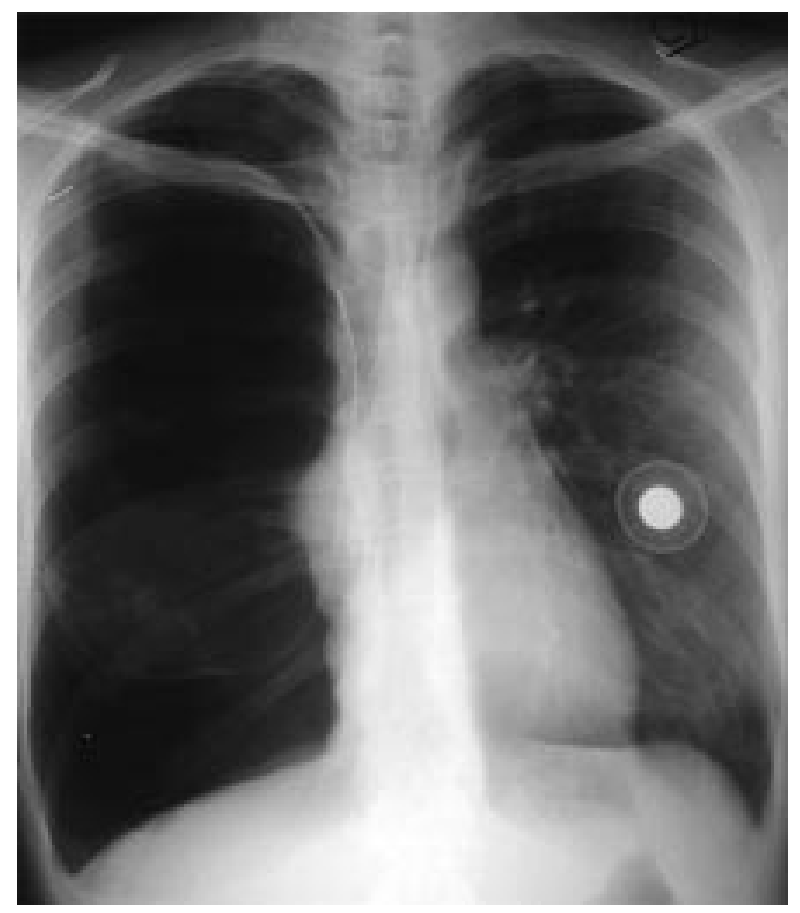

Fig 1. Giant bulla occupying most of right hemithorax.

The resected specimen was $15 \times 10 \times 0.5 \mathrm{~cm}$. It was pinktan and consisted of primarily a giant cystic lesion, which was thick walled, and a small amount of upper lobe. Microscopically, the adjacent lung parenchyma revealed features reminiscent of placental chorionic villi. Areas of fibrosis were admixed with vague papillary structures. The papillae were characterized by vascular channels, pale-staining, cuboidal, stromal cells, mononuclear cells, and smooth muscle bundles (Fig 3).

Discussion. Giant bullae occupying the entire hemithorax are not frequently seen. Severe emphysema usually affects both lungs and is related to smoking. This patient had a bulla that was very large and chronic, causing significant collapse of the middle and lower lobes. This was mistaken for a pneumothorax, which can commonly occur, and resulted in thoracostomy tube placement. Computed tomographic scan was useful in delineating the true pathologic condition.

Placental transmogrification was first described in 1979 as a rare histologic subtype of bullous emphysema and has since been described in 8 patients. ${ }^{1-4}$ The patients ranged in age from 24 to 44 years and have included 6 men and 2 women. Surgical treatment has included lobectomy $(n=5)$ or pneu- 


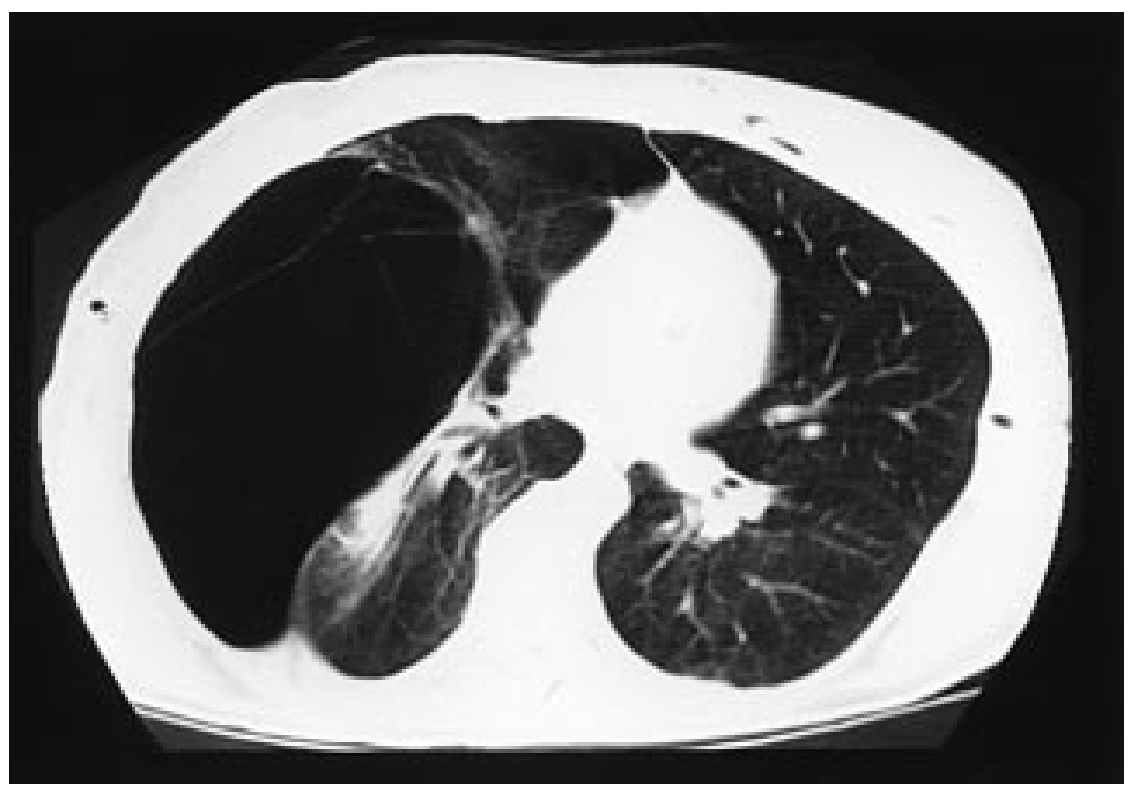

Fig 2. Computed tomographic scan demonstrates right upper lobe bulla with partial collapse of middle and lower lobes.

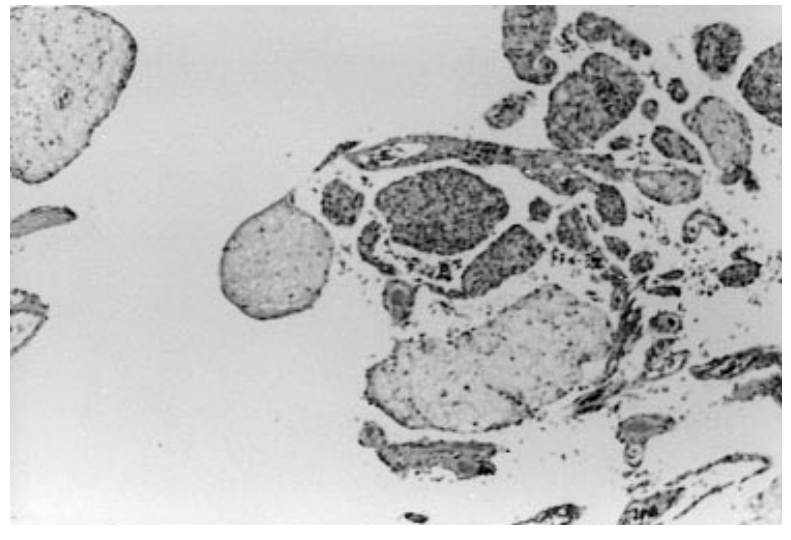

Fig 3. Hematoxylin and eosin section: placental transmogrification of the lung. Giant bulla with features typical of chorionic villi.

monectomy $(\mathrm{n}=3)$, all via thoracotomy. To our knowledge, this report is the first in which thoracoscopy was used. One of the potential limitations of thoracoscopic approach is the limited ability to evaluate the lung tactilely. Subsequent wedge resection was used to remove an additional bulla, preserving the majority of the lower lobe.

The radiologic presentation of placental transmogrification of the lung can be dramatic. Traditionally, anatomic resection has yielded good results. We achieved lung preservation with wedge resection via video-assisted techniques. A basic tenet of this approach is that the staple line be placed through healthy appearing lung. The entire lung should be carefully inspected because more than 1 lobe may be involved.

\section{REFERENCES}

1. McChesney T. Placental transmogrification of the lung: a unique case with remarkable histopathologic features [abstract]. Lab Invest 1979;40:245-6.

2. Horsley WS, Gal AA, Mansour KA. Unilateral giant bullous emphysema with placental transmogrification of the lung. Ann Thorac Surg 1997;64:226-8.

3. Mark EJ, Muller KM, McChesney T, et al. Placentoid bullous lesion of the lung. Hum Pathol 1995;26:74-9.

4. Fidler ME, Koomen M, Sebek B, et al. Placental transmogrification of the lung, a histologic variant of giant bullous emphysema: clinicopathologic study of three further cases. Am J Surg Pathol 1995;19:563-70. 